\title{
Systemic treatment for advanced urothelial cancer: an update on recent clinical trials and current treatment options
}

\author{
Inkeun Park ${ }^{1}$ and Jae Lyun Lee ${ }^{2}$
}

${ }^{1}$ Division of Medical Oncology, Department of Internal Medicine, Gachon University Gil Medical Center, Incheon, ${ }^{2}$ Daparatment of Oncology, Asan Medical Center, University of Ulsan College of Medicine, Seoul, Korea

Received: May 8, 2020

Accepted: June 26, 2020

\section{Correspondence to}

Jae Lyun Lee, M.D.

Daparatment of Oncology, Asan Medical Center, University of Ulsan College of Medicine, 88 Olympic-ro 43-gil, Songpa-gu, Seoul 05505, Korea

Tel: +82-2-3010-5977

Fax: +82-2-3010-6961

E-mail:jaelyun@amc.seoul.kr https://orcid.org/o000-00029420-7162
After cisplatin-based chemotherapy became the standard treatment for metastatic urothelial cancer (mUC), very little progress has been made in the treatment landscape of this condition until recently. With increased knowledge about the molecular biology of mUC and advances in the field of cancer immunobiology, there has been an explosion in the number of clinical trials for mUC, and systemic treatment of mUC is rapidly changing. Despite the availability of several novel therapeutic agents, cisplatin-based cytotoxic chemotherapy remains the standard, first-line treatment option. Immune checkpoint inhibitors (ICIs), including programmed death-1 and programmed death ligand-1 inhibitors, are preferred second-line treatment options that are also used in first-line cisplatin-ineligible settings. For patients with actionable fibroblast growth factor receptor 2 (FGFR2) or FGFR3 genomic alterations, erdafitinib can be considered after platinum-based treatment. Enfortumab vedotin, a monoclonal antibody targeting nectin-4 conjugated to monomethyl auristatin E, has been approved for patients who do not respond to both cytotoxic chemotherapy and ICIs. In this review, we address the clinical trial data that have established the current standard treatments and ongoing clinical trials of various agents with different mechanisms as well as provide a brief overview of current practice guidelines and recommendations in patients with mUC.

Keywords: Carcinoma, transitional cell; Therapeutics; Drug therapy; Immunotherapy

\section{INTRODUCTION}

Bladder cancer is the 1oth most common cancer worldwide, with an estimated 549,000 new cases and 200,000 deaths [1]. Urothelial cancer (UC) is the most common histology among cancers arising from the epithelium lining the urinary tract, any part of the renal pelvis, ureter, urinary bladder, and urethra [2]. Approximately $75 \%$ of patients with urothelial bladder cancer are diagnosed with non-muscle-invasive bladder cancer (NMIBC), which is usually managed by repeated local treatment with surveillance and is considered non-fatal. However, $25 \%$ of patients with bladder cancer have muscle-invasive bladder cancer (MIBC), which requires a multidisciplinary approach, including radical cystectomy and perioperative chemotherapy, and some of them have locally advanced unresectable, recurrent, or metastatic disease [3]. The prognosis of metastatic urothelial cancer (mUC) is poor, with a median overall survival (mOS) of approximately 15 months in large randomized trials [4,5]; 
the 5-year survival rates remained stable at approximately $10 \%$ during 1973 to 2005 [6].

Until mid-2010, there had been little progress in systemic treatments for mUC because of the wide usage of platinum-based combination chemotherapies in metastatic disease since the 1980s. However, mUC is one of the most rapidly progressing fields in medical oncology, with the recognition of actionable molecular alterations and introduction of novel agents, including immune checkpoint inhibitors (ICIs), molecularly targeted agents (MTAs), and antibody-drug conjugates (ADCs). In this review, we address the clinical trial data that have established the current standard treatments and ongoing clinical trials of various agents with different mechanisms, and also provide a brief overview of current practice guidelines and recommendations in patients with mUC.

\section{CYTOTOXIC CHEMOTHERAPY}

\section{First-line cisplatin-based chemotherapy}

Cytotoxic chemotherapy has been the mainstay of systemic treatment for $\mathrm{mUC}$, and objective response rates (ORRs) to contemporary first-line combination cytotoxic chemotherapies range from $50 \%$ to $65 \%$ with complete response (CR) rates of $10 \%$ to $20 \%[7,8]$. Among various chemotherapeutic agents, cisplatin has been the backbone of treatment, based on ORRs of approximately $33 \%$ as a single agent $[9,10]$. Thereafter, combination regimens containing cisplatin were developed. In a phase II trial, methotrexate, vinblastine, adriamycin, and cisplatin (MVAC) showed a promising response rate of $70 \%$ with an mOS of 13 months [11]. Subsequently, in two randomized phase III trials, MVAC outperformed cisplatin monotherapy [12] as well as cisplatin, cyclophosphamide, and adriamycin combination [13] and became a standard treatment for mUC. Despite MVAC being superior to other agents, it is associated with severe toxicities, including grade 3 or 4 leukopenia, thrombocytopenia, febrile neutropenia, sepsis, mucositis, nausea/ vomiting, renal toxicity, hepatic toxicity, and therapy-related deaths $[11,12]$. Therefore, a less toxic regimen with similar or better efficacy was needed.

Gemcitabine demonstrated its efficacy in phase II trials as a second-line monotherapy and a first-line combination with cisplatin $[14,15]$. In a randomized phase III comparing gemcitabine plus cisplatin (GP) to MVAC, GP achieved comparable ORR (49\% for GP vs. $46 \%$ for MVAC), median progression-free survival (mPFS, 7.7 months vs. 8.3 months), and $\operatorname{mOS}$ (14.0 months vs. 15.2 months) $[5,8]$. Although the study failed to prove a statistical difference in overall survival (OS), the primary endpoint, GP became the preferred first-line chemotherapy owing to its favorable toxicity profile. A 4-week schedule of GP was employed in the trial, but a 3-week schedule is widely used because of similar dose intensity and response rates with better compliance profile $[16,17]$.

Clinical trials continued to improve the efficacy of cytotoxic chemotherapy. As taxanes showed moderate efficacy as a single agent $[18,19]$, they were evaluated in combination with cisplatin in randomized phase III trials (docetaxel plus cisplatin vs. MVAC [20] and larotaxel plus cisplatin vs. GP [21]), but failed to prove their superior efficacy. In a randomized phase III trial EORTC 30987, a combination of paclitaxel and GP was compared to GP in patients with locally advanced or metastatic UC. Even though the triplet group had a higher ORR (55.5\% vs. $43.6 \%, p=0.031)_{2}$ the study failed to show a statistically significant difference in OS, the primary endpoint [22].

Intensification of the standard treatments was also evaluated. The EORTC 30924 trial was a phase III randomized controlled trial that compared dose-dense MVAC (ddMVAC) plus prophylactic granulocyte colony-stimulating factor (G-CSF) with classic MVAC [4,7]. Although ddMVAC did not achieve a statistically significant difference in $\operatorname{mOS}$ (15.1 months vs. 14.9 months, $p=0.049)$, ddMVAC showed higher ORR (64\% vs. 50\%) and better long-term survival (22\% vs. $14 \%$ of 5 -year OS rate) with a more favorable toxicity profile $[4,7]$. Therefore, ddMVAC is recommended as a viable option in the treatment of mUC in guidelines [23-25]. Until now, despite considerable efforts, MVAC and GP are the standard first-line treatments for mUC for several decades.

\section{First-line chemotherapy in cisplatin-unfit patients}

Carboplatin is another platinum chemotherapeutic agent, which is devoid of nephrotoxicity, less emetogenic, and less neurotoxic [26]. There have been no adequately powered randomized trials comparing cisplatin- and carboplatin-based chemotherapy in mUC. However, some small studies and meta-analyses suggested that cisplatin-based chemotherapy significantly 


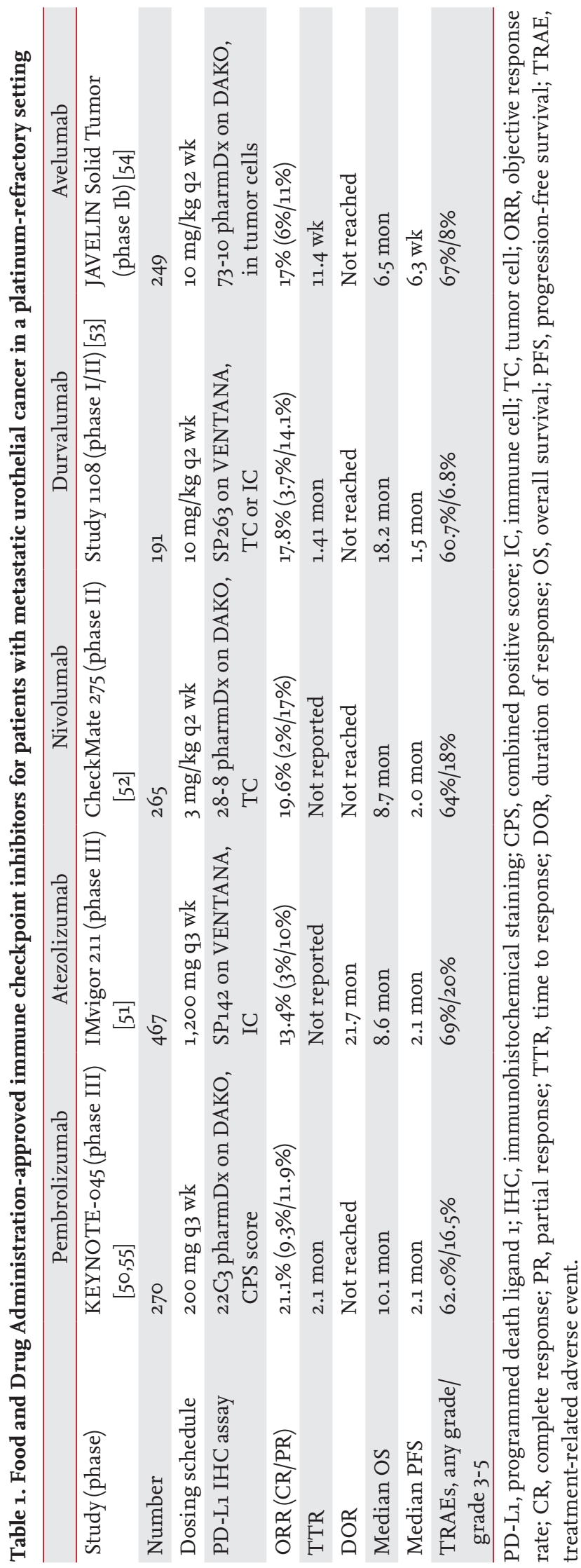

increases the likelihood of achieving a response in mUC [27-29]. Therefore, cisplatin-based chemotherapy is recommended as the first-line treatment in current guidelines, while carboplatin is considered only when patients cannot tolerate cisplatin [23-25]. According to the expert consensus definition, cisplatin "unfit" is defined as the presence of at least one of the Eastern Cooperative Oncology Group performance status 2, creatinine clearance $<60 \mathrm{~mL} / \mathrm{min}$, peripheral neuropathy $\geq$ common terminology criteria of adverse event (CTCAE) v4.o grade 2, hearing loss $\geq$ CTCAE v4.o grade 2, or New York Heart Association class III heart failure [30]. Approximately $30 \%$ to $50 \%$ of patients with $\mathrm{mUC}$ are ineligible to cisplatin in the clinical practice [31]. For platinum unfit patients with $\mathrm{mUC}$, large randomized phase III trials are scarce. EORTC 30986 is the only phase III randomized controlled trial in a platinum unfit population, which compared gemcitabine and carboplatin (GCb) versus methotrexate/carboplatin/vinblastine [32]. The ORR was higher in the GCb arm (41.2\% vs. 30.3\%), but OS and PFS were not significantly different between the two regimens. Although EORTC 30986 failed to prove the superiority of $\mathrm{GCb}$, considering the lower incidence of severe acute toxicity in the GCb arm, GCb became the de facto standard treatment for platinum unfit mUC. Some other doublet combinations were evaluated in phase II trials [33-35], but evidence is insufficient to recommend any specific regimen in this population. Notably, in the COACH trial, gemcitabine plus oxaliplatin maintained its efficacy in patients with very poor renal function (chronic kidney disease stage 4-5), contrary to GCb [34]. Since renal impairment is the most common cause of cisplatin-ineligibility, additional investigation is needed to confirm this finding.

\section{Salvage chemotherapy after failure of first-line treatment}

Although the first-line cisplatin-based chemotherapies have high ORRs, the response does not last long with an mPFS of 7 to 8 months [4,5]. For second-line therapy, many drugs were tested in phase II trials [36-47], but their efficacy was modest, with an ORR of 5-20\%. There has been no successful phase III clinical trial confirming the survival benefit of one salvage chemotherapy regimen over another regimen or best supportive care (BSC). Vinflunine is the only cytotoxic chemotherapeu- 
tic agent that has been tested in a randomized phase III trial. In that trial, vinflunine did not reach statistical significance in improving mOS compared to BSC in the intention-to-treat (ITT) analysis (6.9 months vs. 4.3 months), although the use of vinflunine was independently correlated with improved survival in multivariable analysis $[48,49]$. Until mid-2010, there was no standard salvage treatment, and treatment guidelines recommended various agents such as taxanes (either paclitaxel or docetaxel), vinflunine, or sometimes pemetrexed [23-25].

\section{IMMUNE CHECKPOINT INHIBITORS}

\section{ICls in platinum-pretreated population}

There are five U.S. Food and Drug Administration (FDA)-approved anti-programmed death 1 (PD-1)/programmed death ligand $1(\mathrm{PD}-\mathrm{L} 1)$ antibodies, nivolumab, pembrolizumab, durvalumab, avelumab, and atezolizumab, for mUC (Table 1) [50-54]. Among these drugs, pembrolizumab (KEYNOTE-045) and atezolizumab (IMvigor-211) underwent phase III randomized controlled trials in a salvage setting $[50,51]$. In contrast, others have only phase I or II trial results.

In KEYNOTE-045, the efficacy of anti-PD-1 antibody pembrolizumab as a salvage treatment was compared with the investigator's choice drug (paclitaxel, docetaxel, or vinflunine) in patients with $\mathrm{mUC}$ who had disease progression after platinum-based chemotherapy [50]. Co-primary endpoints were OS and PFS in the total population and in the patients with PD-L1 expressing tumors according to two thresholds (combined positive score $[\mathrm{CPS}] \geq 1$ and $\geq 10$ ). A total of 542 patients were randomly assigned to the pembrolizumab and chemotherapy groups, and in the second interim analysis, co-primary endpoints were met. The mOS was 10.3 months with pembrolizumab and 7.4 months with chemotherapy (hazard ratio [HR], $0.73 ; p=0.002$ ). There was no significant difference in mPFS (2.1 months vs. 3.3 months). ORR was significantly higher in pembrolizumab (21.1\% vs. $11.4 \%, p=0.001$ ). Long-term efficacy and safety results were consistent with the interim results [55].

IMvigor211 was a randomized phase III trial comparing atezolizumab, an anti-PD-L1 antibody with the investigator's choice drug (paclitaxel, docetaxel, or vinfl- unine) [51]. Eligibility criteria were generally similar to those of KEYNOTE-045. The primary endpoint was OS tested hierarchically in prespecified populations; $\mathrm{PD}-\mathrm{L} 1$ expression on $\geq 5 \%$ of tumor-infiltrating immune cells (IC2/3), followed by IC1/2/3 (PD-L1 expression on $\geq 1 \%$ of immune cells), followed by the ITT population. A total of 931 patients were randomized to the atezolizumab or chemotherapy arm. In the IC2/3 population $(n=234)$, mOS did not differ significantly between patients in the atezolizumab group and in the chemotherapy group (11.1 months vs. 10.6 months; HR, $0.87 ; p=0.41$ ), precluding further formal statistical comparisons. The ORR was similar between treatment arms (23.0\% vs. $21.6 \%$ in IC2 $/ 3$ population and $13.4 \%$ vs. $13.4 \%$ in ITT population), while mPFS was numerically shorter in the atezolizumab arm (2.4 months vs. 4.2 months in the IC2/3 population and 2.1 months vs. 4.0 months in the ITT population). Therefore, in contrast to the KEYNOTE-O45 trial, IMvigor211 failed to prove the efficacy of atezolizumab over cytotoxic chemotherapy. However, in exploratory analysis of the ITT population of IMvigor211, the atezolizumab arm had a numerically improved OS compared to the chemotherapy arm. The mOS was 8.6 months versus 8.0 months, and the 1-year OS rate was $39.2 \%$ versus $32.4 \%$, with an HR, 0.85 (95\% confidence interval [CI], 0.73 to 0.99). The atezolizumab arm maintained the improved OS in an updated analysis, demonstrating a 2-year OS rate of $23 \%$ versus $13 \%$ and a 3 -year OS rate of $18 \%$ versus 10\% with an HR of 0.82 (95\% CI, 0.71 to 0.94 ) [56]. This unexpected finding gives us a lesson that design and statistical analysis plan are important in clinical trials, especially when biomarkers, which have not been fully understood, are incorporated into trials. Based on these results, pembrolizumab became the standard treatment for platinum-pretreated mUC. However, as there is no clinical trial conducting head-to-head comparison of various ICIs in mUC, it is not evident whether pembrolizumab is superior to other ICIs. Considering real-world outcomes of ICIs were similar to those of KEYNOTE-O45 or IMvigor211 [57,58], atezolizumab or other ICIs can also be used in platinum-pretreated populations [24].

The incidence of treatment-related adverse events (AEs) of ICIs was lower than that of cytotoxic chemotherapy. For instance, any grade and grade 3-5 AEs occurred in $60.9 \%$ and $15.0 \%$, respectively, of patients in the pembrolizumab arm, compared to $90.2 \%$ and $49.4 \%$, 
990 KEYNOTE-361 (NCT02853305)

First-line unresectable/metastatic UC

Co-primary endpoints: PFS and OS

Estimated study period: Sep 2016-May 2020

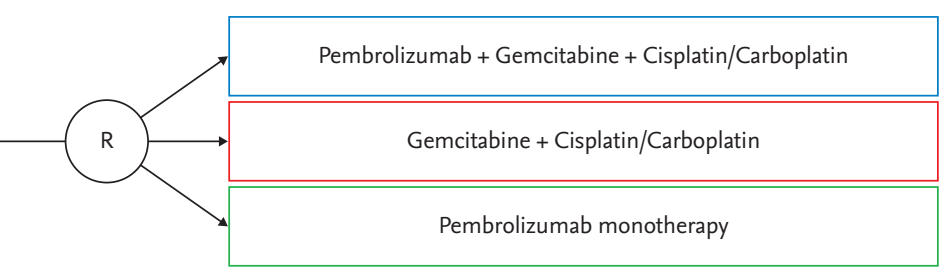

990 Check Mate-901 (NCT03036098)

Fist-line unresectable or metastatic UC

Co-primary endpoints: OS in cisplatin-ineligible participant and

OS in $\mathrm{PD}-\mathrm{Ll}(+)$ participant

Estimated study period: Mar 2018-Dec 2022
1,200 IMvigor130 (NCT02807636)

Fist-line platinum eligible locally advanced or metastatic UC

Estimated study period: Jun 2016-Nov 2020
Co-primary endpoint: PFS, OS, and safety
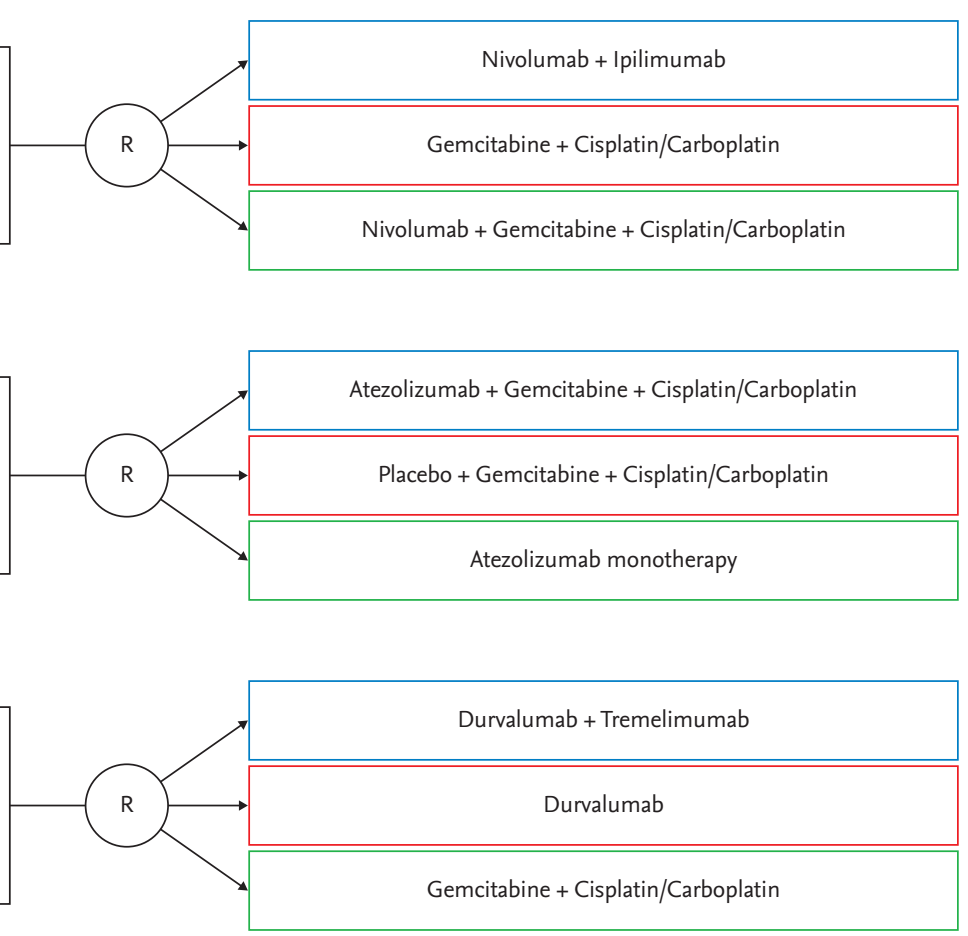

126 (actual enrollment), DANUBE (NCT02516241)

First line unresectable or metastatic UC

Estimated study period: Nov 2015-May 2020
885 NILE (NCT03682068)

First line unresectable or metastatic UC

Co-primary endpoints: PFS and OS

Estimated study period: Sep 2018-Apr 2022

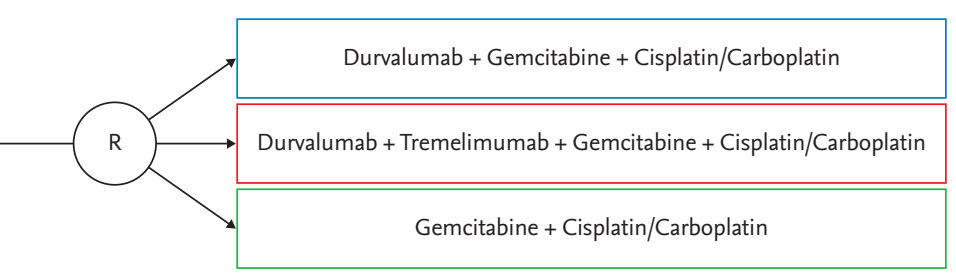

694 LEAP-011 (NCT03898180)

First line unresectable or metastatic UC

Cisplatin-ineligible with PD-LI CPS $\geq 10$ or platinum-ineligible

Co-primary endpoints: PFS and OS

Estimated study period: May 2019-Dec 2022

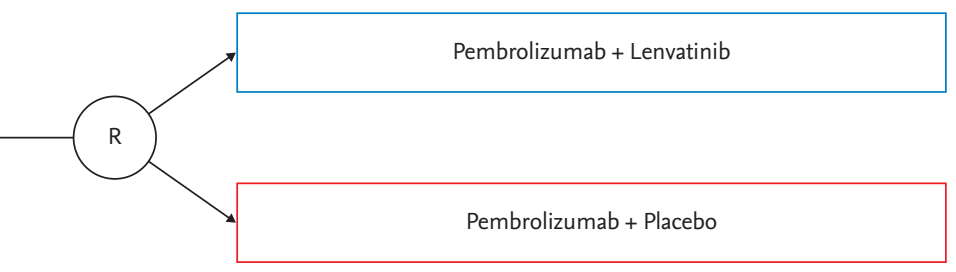

Figure 1. Ongoing first-line phase III trials investigating immune checkpoint inhibitors in advanced urothelial cancer. UC, uorthelial carcinoma; PFS, progression-free survival; OS, overall survival; PD-L1, programmed death ligand-1; CPS, combined positive score.

respectively, of patients in the chemotherapy arm in the KEYNOTE-045 trial [50]. Likewise, any grade and grade
3-5 AEs were reported in 69\% and 20\%, respectively, of patients in the atezolizumab arm, compared to $89 \%$ and 
$43 \%$, respectively, of patients in the chemotherapy arm in the IMvigor211 trial [51]. The most common AEs for ICIs are pruritus, fatigue, nausea, diarrhea, decreased appetite, and rash. Although not typical, there are immune-related AEs that could sometimes be fatal. Thus, physicians need to be aware of the immune-related AEs and management $[59,60]$. In addition, there are distinct radiologic response and progression patterns of ICIs, such as pseudoprogression and hyperprogression [61]. The exact incidences of these phenomena in UC are not known, but these might be not uncommon, as $1.5 \%$ to $17 \%$ of pseudoprogression and $12 \%$ of hyperprogression are reported in mUC $[62,63]$.

\section{Maintenance $\mathrm{ICl}$ after first-line chemotherapy}

Previous studies have investigated the efficacy of ICIs in mUC patients who experienced disease progression during or after platinum-based chemotherapy. In contrast, a phase III randomized trial, JAVELIN Bladder 100 trial (NCTo2603432) investigated maintenance treatment with avelumab plus BSC versus BSC alone in patients with mUC whose disease did not progress after completion of first-line platinum-containing chemotherapy, and primary analysis results were presented at the American Society of Clinical Oncology meeting in 2020 [64]. A total of 700 patients were randomized, and a statistically significant improvement in OS was demonstrated in the avelumab arm in both ITT group (21.4 months vs. 14.3 months; stratified HR, 0.69 [95\% CI, 0.56 to 0.86 ]; $p<0.001$ ) and PD-L1 positive group (not reached vs. 17.1 months; stratified HR, 0.56 [95\% CI, 0.40 to 0.79]; $p<0.001$ ) [64]. Both PFS and ORR were also superior in the avelumab arm. Based on these results, avelumab maintenance in mUC patients whose disease has not progressed with first-line platinum-based chemotherapy would be a new standard of care.

\section{First-line $\mathrm{ICl}$ in cisplatin-unfit patients}

In cisplatin-unfit patients, GCb has been a de facto standard treatment after the EORTC 30986 trial [32], but there is an urgent need to improve treatment in this population. In IMvigor 210 (cohort 1) and KEYNOTE-052 trials, atezolizumab and pembrolizumab were tested as monotherapy in cisplatin-unfit chemotherapy-naïve patients with mUC [65-67]. In the IMvigor 210 trial, out of 123 patients who received atezolizumab, ORR, mPFS, and mOS were 23\%, 2.7 months, and 15.9 months, respectively [65]. In the KEYNOTE-052 trial, out of 370 patients, ORR, mPFS, and mOS were 29\%, 2.2 months, and 11.3 months, respectively $[66,67]$. Notably, patients with $\mathrm{PD}-\mathrm{L} 1 \mathrm{CPS} \geq 10$ achieved more favorable outcomes with pembrolizumab compared to CPS $<10$, with an ORR of $47.3 \%$ versus $20.3 \%$ and an mOS of 18.5 months versus 9.7 months in KEYNOTE-052. Based on these results, both atezolizumab and pembrolizumab were granted accelerated approval by the U.S. FDA.

For continued approval, confirmatory data in phase III trials are required from IMvigor 130 (NCTo2807636) and KEYNOTE-361 (NCTo2853305) trials. In the preliminary analyses of both trials, in patients with low PD-L1 expression, ICI arms had decreased survival compared to cisplatin- or carboplatin-based therapy. As a result, both trials stopped enrollment of patients with low PDL1 status into monotherapy arms, and the indication for both agents was modified to include only patients who were not eligible for cisplatin-containing chemotherapy and who had high expression of PD-L1 or were not eligible for any platinum-containing chemotherapy regardless of the level of PD-Li expression [68]. In the interim OS results of the IMvigorızo trial [69], although atezolizumab monotherapy group had numerically longer mOS than the chemotherapy group (15.7 months vs. 13.1 months), it was difficult to draw any conclusion because the stratified HR of 1.02 (95\% CI, 0.83 to 1.24) and crossing survival curves suggested heterogeneous treatment effect across subgroups. Furthermore, formal statistical testing for comparing atezolizumab monotherapy with chemotherapy was not performed because of the hierarchal statistical design of the trial.

\section{Ongoing first-line combination ICI trials}

There are multiple ongoing clinical trials in the firstline setting, investigating whether the combination of ICIs with cytotoxic chemotherapy is superior to chemotherapy alone or ICI alone (Fig. 1). In addition, a clinical trial is evaluating first-line pembrolizumab in combination with lenvatinib versus pembrolizumab plus placebo in cisplatin-ineligible patients with CPS $\geq 10$ or platinum-ineligible patients (LEAP-o11 trial, NCTo3898180). Among these trials, the final PFS and interim OS results of the IMvigor130 trial have been published [69]. After a median follow-up of 11.8 months, the mPFS was sig- 


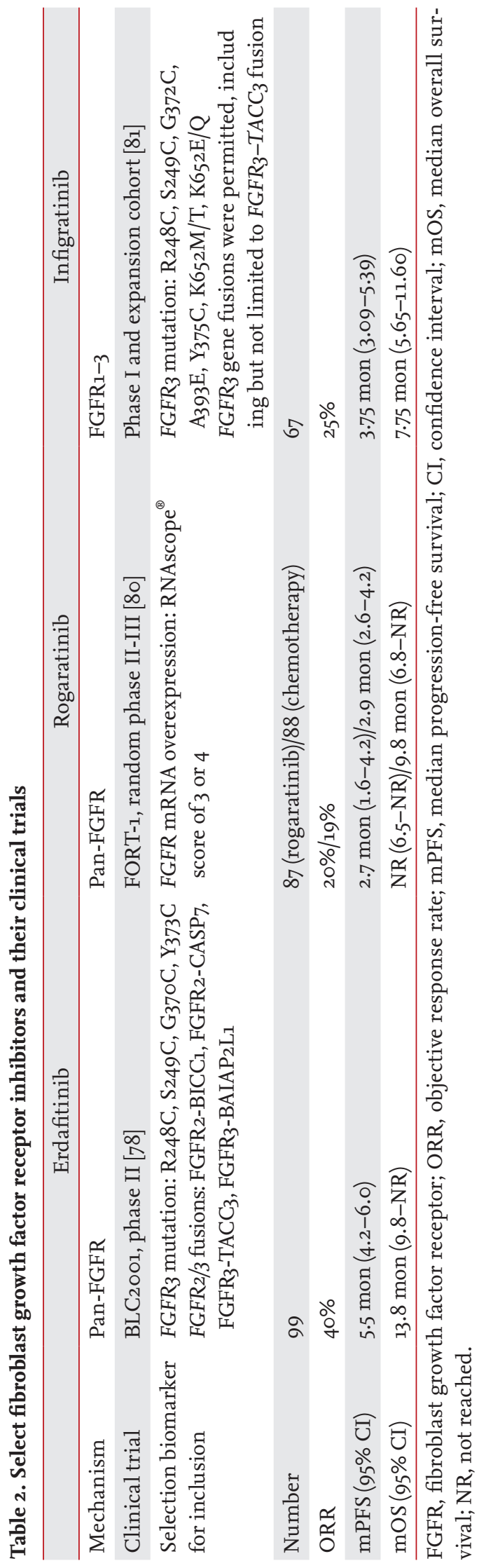

nificantly superior in the combination arm: 8.2 months in the chemotherapy plus atezolizumab arm versus 6.3 months in the chemotherapy plus placebo arm (HR, 0.82; $95 \% \mathrm{CI}, 0.70$ to $0.96 ; p=0.007$ ). $\mathrm{mOS}$ was 16.0 months versus 13.4 months, with a stratified HR of 0.83 (95\% CI, 0.69 to 1.00) and a one-sided $p$ value of 0.027 . As the $p$ value did not exceed the prespecified interim efficacy boundary, further follow-up for mature OS data is required. In addition, in the DANUBE trial, the primary endpoints were not attained [70]. A full report of the DANUBE trial has not been published yet.

\section{MOLECULARLY TARGETED AGENTS}

Owing to next-generation sequencing and bioinformatics, genomic landscape and actionable mutations in UC have been unveiled [71-74]. According to the updated results of The Cancer Genome Atlas (TCGA) report [74], the most common mutations in MIBC are TP53, PIK${ }_{3} C A, C D K N 1 A, E R C C 2$, fibroblast growth factor receptor 3 (FGFR3), and ERBB3. Most recently conducted trials have focused on actionable mutations among the aforementioned alterations.

\section{Targeting fibroblast growth factor receptor}

FGFR3 alteration is a common event in UC, with a spectrum of mutations, including point mutation and fusion, having been reported [75]. Although FGFR3 alterations are more common in NMIBC (up to $80 \%$ in Ta and $30 \%$ in $\mathrm{T} 1$ ) [76], $12 \%$ to $15 \%$ of MIBC patients have FGFR3 alterations [71-74]. Activating point mutations in exons 7, 10, and ${ }_{15}$ (S249C, R248C, and $\mathrm{Y}_{373} \mathrm{C}\left[\mathrm{Y}_{375} \mathrm{C}\right]$ ) are the most common FGFR3 alterations, and less commonly, gene fusions (FGFR3-TACC 3 , FGFR3-BAIAP2L1, and FGFR3-JAKMIP1) have also been observed [75]. In addition to mutations, FGFR3 protein or mRNA overexpression is also present in MIBC [77], probably by epigenetic regulation [78]. As FGFR3 alteration plays an important oncogenic role in UC [75], FGFR3 signaling is an attractive target, and many drugs have been tested in UC [7985]. The outcomes of select trials of FGFR inhibitors in mUC are described in Table 2, and ongoing trials of MTAs are listed in Table 3.

Erdafitinib is a potent tyrosine kinase inhibitor (TKI) of FGFR1-4. In the BLC2001 trial, a phase II trial eval- 


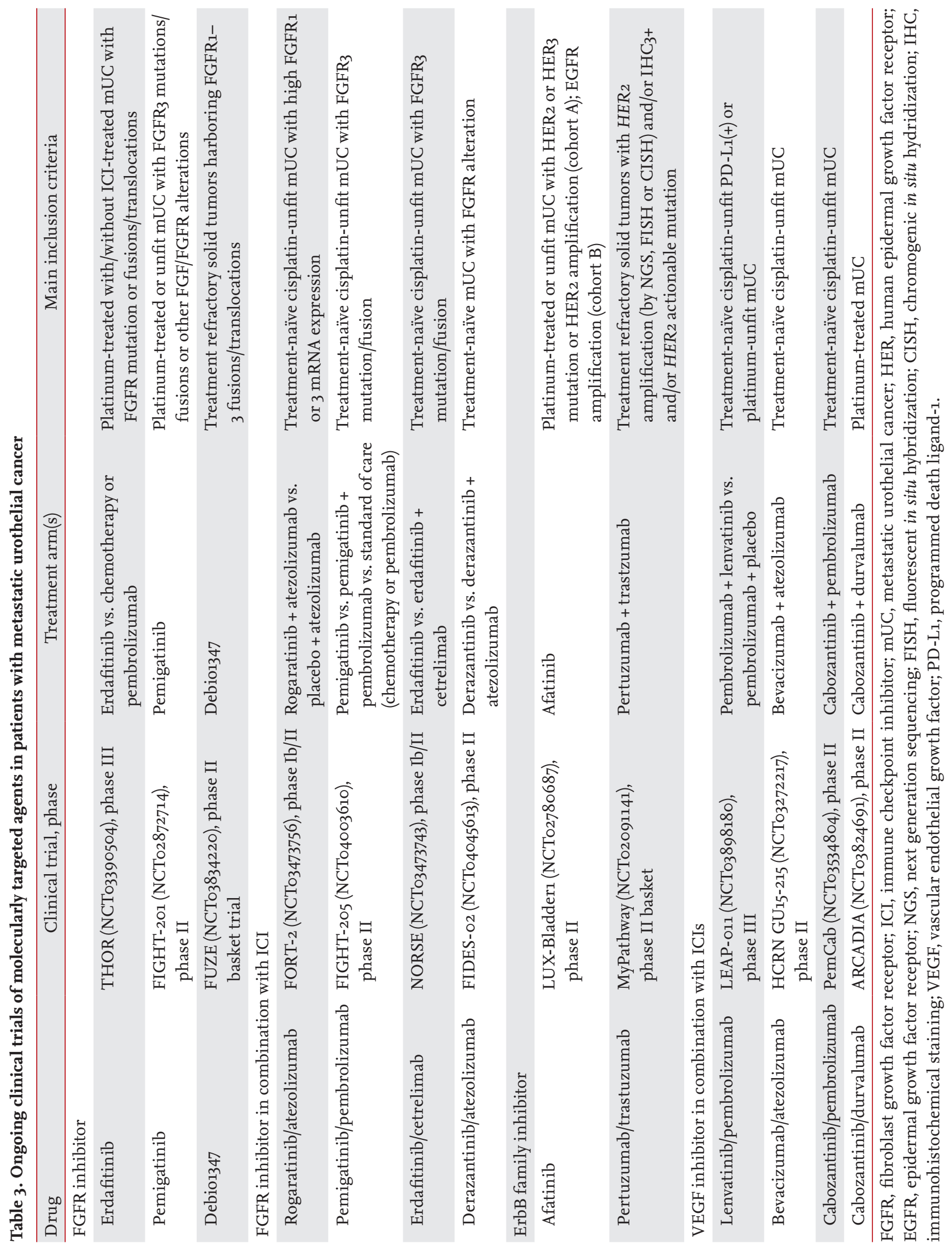


uating erdafitinib in chemotherapy-refractory or unfit patients with $\mathrm{mUC}$ with susceptible FGFR alterations (FGFR3 mutation or FGFR2/3 fusion), patients were treated with a daily dose of erdafitinib [8o]. In the selected-regimen population of 99 patients, the confirmed ORR was $40 \%$, and $\mathrm{mPFS}$ and mOS were 5.5 months (95\% CI, 4.2 to 6.0.) and 13.8 months (95\% CI, 9.8 to not reached), respectively. Based on this result, erdafitinib received FDA approval for the treatment of advanced UC with susceptible FGFR3 or FGFR2 alteration. A confirmatory randomized phase III THOR trial (NCT03390504) for comparing erdatifinib with chemotherapy (vinflunine or docetaxel) or pembrolizumab in patients with FGFR mutations or fusions/translocations is ongoing (Table 3).

FGFR pathway activation is associated with non-T-cell inflamed tumors in MIBC [86], which means that ICI might be less effective in FGFR-activated mUC. There is preclinical and clinical evidence that FGFR inhibition increases $\mathrm{T}$ cell infiltration and reduces Tregs, and upregulates genes associated with inflammatory responses $[87,88]$. Against this background, several trials evaluating the combination of FGFR inhibitors and immunotherapeutics were initiated. Some of these were reported $[88,89]$, and others are ongoing (Table 3$)$.

\section{Targeting the ErbB family (EGFR, HER2, and HER3)}

The ErbB family of interest in mUC includes epidermal growth factor receptor (EGFR), human epidermal growth factor receptor 2 (HER2), and HER3. EGFR expression is associated with higher grade and stage and poorer prognosis in UC [90]. Anti-EGFR therapies have been tried in mUC, but EGFR TKI gefitinib and anti-EGFR monoclonal antibody cetuximab have shown limited efficacy, regardless of whether it was a monotherapy or a combination with cytotoxic chemotherapy and a salvage treatment, or the front-line treatment [91-94].

There have been a series of studies on the negative prognostic value of HER2 expression in UC [90]; moreover, the TCGA data revealed that $16 \%$ and $12 \%$ of patients with MIBC had HER2 and HER3 alterations (mutation and amplification), respectively [72,74]. Lapatinib, a TKI blocking both EGFR and HER2, has been evaluated in various clinical settings in UC; however, it failed to demonstrate any efficacy [95-98]. Afatinib, a pan-HER inhibitor, also did not meet the primary end- point in a phase II trial [99]. However, considering that all patients who achieved 3-month PFS had HER2 and/ or HER3 alterations in this trial, a phase II trial of afatinib in molecularly selected patients with HER2 or HER3 alteration is underway (NCTo2780687) (Table 3). Trastuzumab is an immunoglobulin $\mathrm{G} 1$ (IgGi) monoclonal antibody targeting HER2 and has been tested in two phase II trials for HER2-overexpressing mUC, as a combination with gemcitabine, carboplatin, and paclitaxel in patients with chemotherapy-naïve mUC [100] and as a combination with GP (GP with vs. without trastuzumab) [101]. Neither trial demonstrated clinically meaningful activity of trastuzumab in mUC. There is an ongoing genomic biomarker-driven basket trial (MyPathway) that includes HER2-directed therapy with pertuzumab plus trastuzumab for patients with mUC with HER2 amplification (by next-generation sequencing, fluorescence in situ hybridization, or chromogenic in situ hybridization) and/or immunohistochemical (IHC) $3+$ and/or HER2 actionable mutation (Table 3) [102].

\section{Targeting vascular endothelial growth factor signaling} Bladder cancer produces pro-angiogenic factors, including vascular endothelial growth factor (VEGF), and high expression of pro-angiogenic factors were found to be correlated with disease progression and poor survival [103]. Several VEGF receptor TKIs have been investigated in mUC, as a monotherapy in phase II trials [104-109] or as a combination with cytotoxic chemotherapy [110-112]. All these agents were not investigated further owing to a lack of efficacy and/or excessive toxicities.

Monoclonal antibodies targeting VEGF signaling were also tested. Ramucirumab, a monoclonal antibody to VEGFR-2, was evaluated in a phase III randomized controlled trial (RANGE) comparing the efficacy of ramucirumab plus docetaxel with placebo plus docetaxel in previously treated mUC $[113,114]$. Bevacizumab, a monoclonal antibody to VEGF-A, was investigated in a phase III randomized controlled trial (CALGB90601) comparing the efficacy of GP plus bevacizumab with GP plus placebo in chemotherapy-naïve patients with mUC [115]. Both trials showed that monoclonal VEGF antibodies combined with cytotoxic chemotherapy failed to improve OS in patients with $\mathrm{mUC}$, although the combinations prolonged PFS.

Besides the antiangiogenic effect, there is preclin- 
ical evidence that VEGF inhibition also facilitates anti-tumor immunity [116]. VEGF inhibition enhances T cell infiltration and activation and inhibits suppressive immune cells. Therefore, the combination of VEGF inhibitors and ICIs can be synergistic, and there are several ongoing clinical trials evaluating the efficacy of a combination of VEGF inhibitors and ICIs in mUC (Table 3).

\section{ANTIBODY-DRUG CONJUGATES}

ADCs are a novel class of drugs that are rationally designed to deliver effective cytotoxic drugs directly and selectively to cancer cells. ADCs comprise a monoclonal antibody that recognizes tumor-associated antigens and to which a potent cytotoxic agent is conjugated via chemical linkages [117]. There are several promising ADCs under evaluation for mUC.

\section{Enfortumab vedotin (ASG-22CE; ASG-22ME)}

Enfortumab vedotin is an ADC that comprises a fully human monoclonal antibody targeting nectin-4 conjugated to monomethyl auristatin E (MMAE) via a protease-cleavable linker [118]. Nectin-4 is a member of the transmembrane protein nectin family cell adhesion molecules that are involved in various cellular processes, including carcinogenesis [119]. Nectin-4 is highly expressed in various solid tumors including UC [120]. In a phase 2, open-label, single-arm study (EV-201), the efficacy of enfortumab vedotin was tested in patients with mUC who previously received an ICI with or without prior platinum chemotherapy. Results of patients who received both ICI and chemotherapy were published [118]. The ORR was $44 \%$ and CR was $12 \%$; mPFS and mOS were 5.8 and 11.7 months, respectively. Treatment response was observed in all subgroups, including ICI non-responders and those with liver metastases. Common treatment-related AEs included fatigue (50\%), peripheral neuropathy (50\%), alopecia (49\%), rash (48\%), decreased appetite (44\%), and dysgeusia (40\%). Grade 3 or more AEs occurred in over $5 \%$ of patients, with only fatigue occurring in $6 \%$. No fatal treatment-related AEs were reported. Based on these data, the FDA approved enfortumab vedotin for mUC following chemotherapy and ICI treatment. Currently, a randomized phase 3 tri- al (EV-301, NCTo3474107) is ongoing to compare enfortumab vedotin with the investigator's choice (docetaxel, paclitaxel, or vinflunine) in patients with mUC who showed disease progression with platinum-based chemotherapy and an ICI (Table 4).

Another ongoing study (EV-103, NCT03288545) is a phase $1 \mathrm{~b}-2$ trial evaluating enfortumab vedotin combined with pembrolizumab and/or chemotherapy in patients with metastatic UC in multiple cohorts. The preliminary results of cohort A, in which 45 cisplatin-ineligible patients were treated with enfortumab vedotin plus pembrolizumab showed that the ORR was 73.3\% with $15.6 \% \mathrm{CR}$ and the response was not associated with PD-L1 status [121]. Based on this promising result, a phase 3 study, EV-302 (NCTo4223856) is designed and currently recruiting patients. This trial will evaluate first-line enfortumab vedotin in combination with pembrolizumab with or without chemotherapy versus chemotherapy in patients with advanced UC.

\section{Sacituzumab govitecan (IMMU-132)}

Sacituzumab govitecan is an ADC in which $\mathrm{SN}-38$ (an active metabolite of irinotecan) is conjugated to the humanized anti-trophoblast cell-surface antigen 2 (Trop2) monoclonal antibody via a cleavable linker. Trop-2, a transmembrane calcium signal transducer, is overexpressed in many epithelial cancers [122], and its expression correlates with disease severity in UC [123].

In a phase I/II basket trial, sacituzumab govitecan was assessed in a UC cohort that included patients with mUC who progressed after one or more prior systemic therapy [124]. In the cohort of 45 patients, the ORR was $31 \%$ with two patients with CR, and the MPFS and mOS were 7.3 and 18.9 months, respectively. Grade 3 or more AEs observed were neutropenia (38\%), anemia (11\%), hypophosphatemia (11\%), diarrhea (9\%), fatigue (9\%), and febrile neutropenia (7\%). TROPHY-U-o1 (NCT03547973) is an open-label, single-arm, phase 2 trial evaluating sacituzumab govitecan in 140 patients with $\mathrm{mUC}$ who failed both platinum-based chemotherapy and ICI (cohort 1,100 patients) or failed ICI in cisplatin-ineligible patients (cohort 2, 40 patients). In pre-planned interim analysis in cohort $1(n=35)$ [125], the ORR was $29 \%$, which surpassed the prespecified futility endpoint; therefore, further enrollment is being continued (Table 4). 


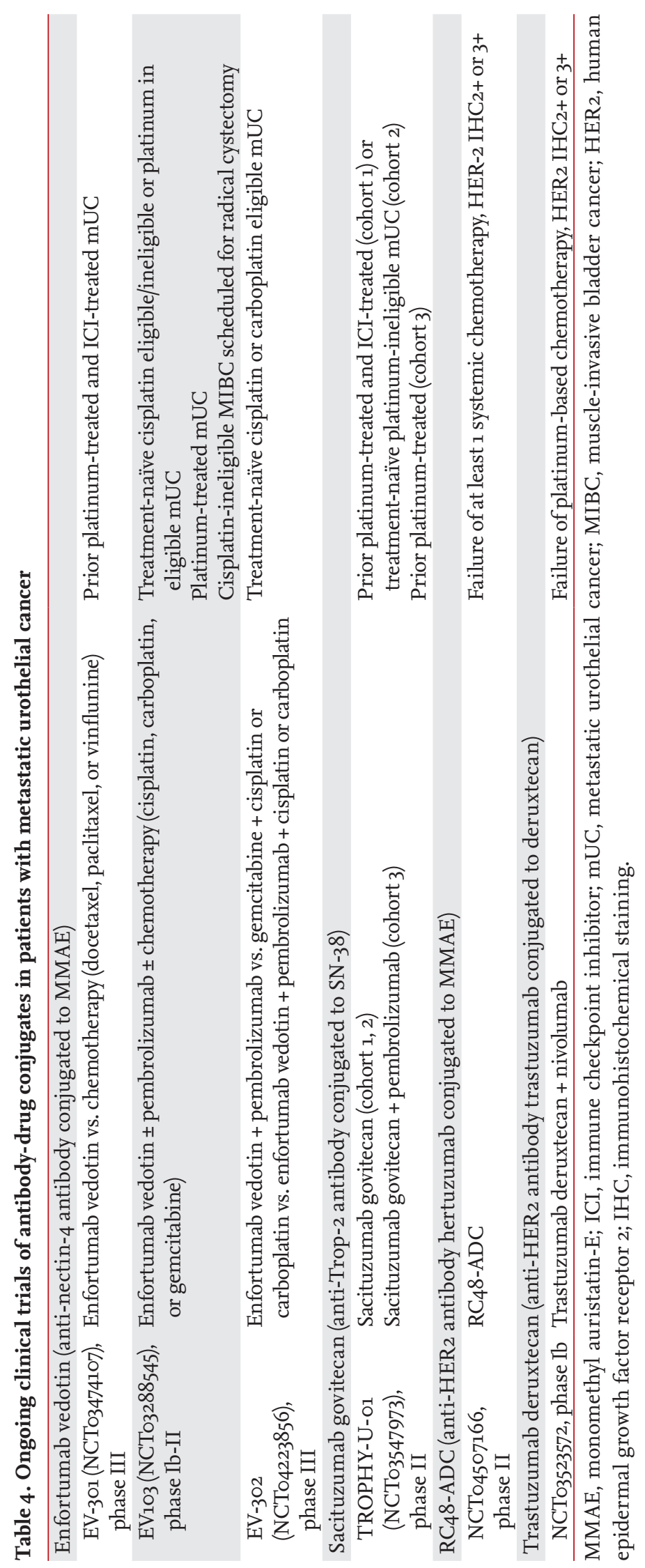

\section{Other ADCs actively investigated in mUC}

Other ADCs under evaluation in $\mathrm{mUC}$ are listed in Table 4. RC48-ADC is a novel humanized high-affinity anti-HER2 antibody hertuzumab conjugated with MMAE via a cleavable linker [126]. A phase II trial (NCT03507166) of HER2-overexpressed (IHC2+ or 3+) pretreated advanced UC completed accrual, and its result is awaited [127]. Trastuzumab deruxtecan (DS8201a) is another trastuzumab-based ADC linked to deruxtecan, a derivative of topoisomerase I exatecan. The phase $1 \mathrm{~b}$ trial combining DS-8201a with nivolum$\mathrm{ab}$ in advanced HER2-expressing breast or UC in underway (NCT03523572) [128].

\section{TREATMENT RECOMMENDATIONS AND SUGGESTED TREATMENT SEQUENCE}

\section{Pre-treatment evaluation}

Before starting the systemic treatment of patients with recurrent or metastatic UC, there are several aspects that must be considered. UC is primarily a disease occurring in elderly individuals. The median age of newly diagnosed patients is 73 years, and most patients are over 65 years [129]. A substantial proportion of this elderly population is excluded from clinical trials; thus, direct extrapolation of clinical trial results into this population can be misleading. Generally, elderly patients experience toxicities more frequently and severely, and the degree of benefit from treatment might be less [130]. For example, in an observational study of real-world practice involving elderly patients with newly diagnosed advanced UC, $42 \%$ of patients received firstline chemotherapy and only $27 \%$ of first-line-treated patients received cisplatin-based chemotherapy, showing a mOS of 8.5 months [131]. In contrast, for a well-selected population, chemotherapy can be similarly effective between young and elderly patients [132]. Therefore, to avoid excessive toxicity and to select potential beneficiaries, a thorough examination, including geriatric assessment is recommended for the elderly population [133]. Furthermore, patients with UC usually have multiple comorbidities. In one study, patients with bladder cancer had a median of 8 (interquartile range, 5 to 11) chronic conditions, including chronic kidney disease, coronary artery disease, and diabetes mellitus [134]. Co- 


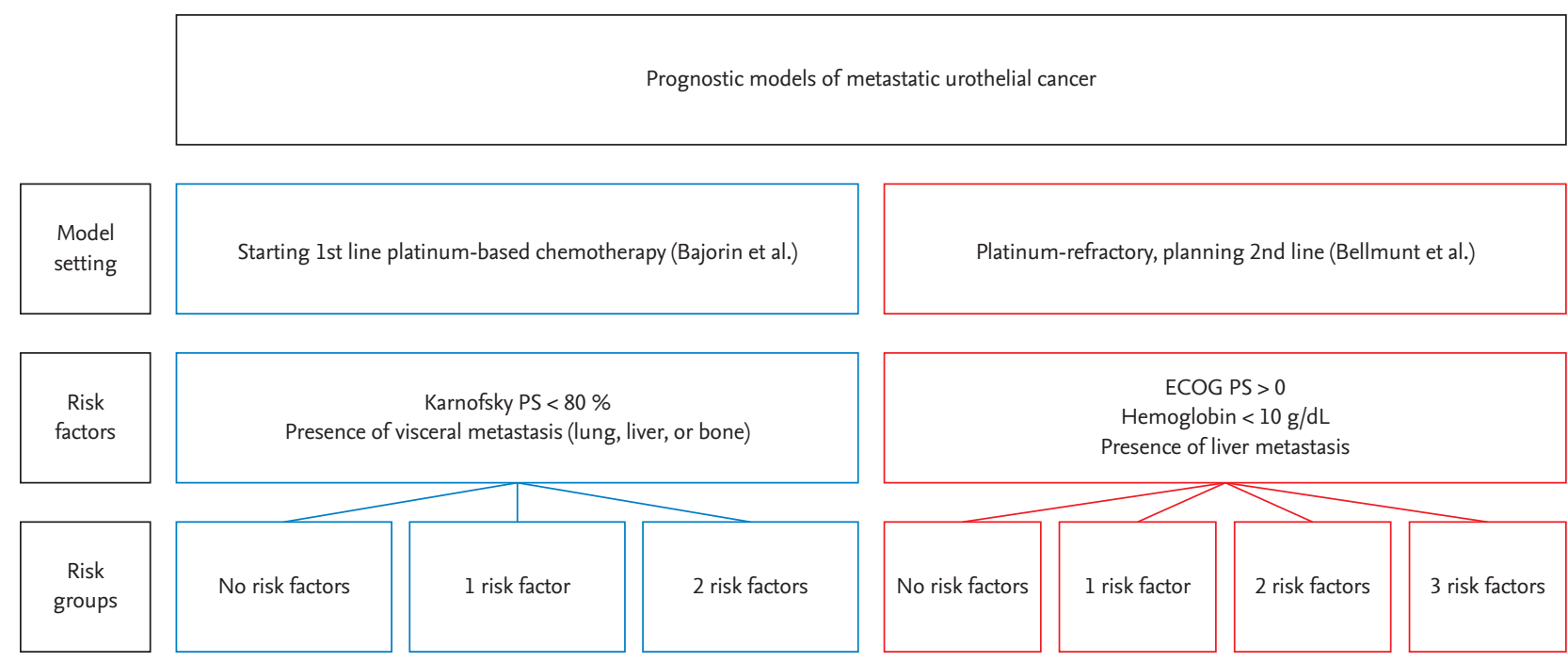

Figure 2. Validated prognostic models in metastatic urothelial cancer. PS, performance status; ECOG, Eastern Cooperative Oncology Group.

morbidities are important in determining the "fitness" for cisplatin-based chemotherapy and seem to affect prognosis [135]. Besides age and comorbidities, socioeconomic status is another important factor affecting chemotherapy administration [136].

Although ICIs are generally well-tolerated and have more favorable toxicity profiles than cytotoxic chemotherapy, there are multiple conditions that need special concern, including autoimmune diseases, ongoing immunosuppressant use, or chronic viral infection. These populations can be cautiously treated with ICIs [137], but shared decision-making and monitoring is required.

\section{Prognostic factors for mUC}

Well-established prognostic models have essential roles in the management of patients with cancer; they enable physicians to predict life expectancy, guide treatment selection, analyze results of clinical studies, and educate patients and their families. There are several prognostic models available for mUC and are used in different clinical situations (Fig. 2).

For patients receiving first-line cisplatin-based chemotherapy, the Bajorin prognostic model can be applied [138]. This model has also been validated in patients receiving first-line carboplatin-based treatment [32]. Additionally, in platinum-refractory settings, the Bellmunt prognostic model can be used [139].

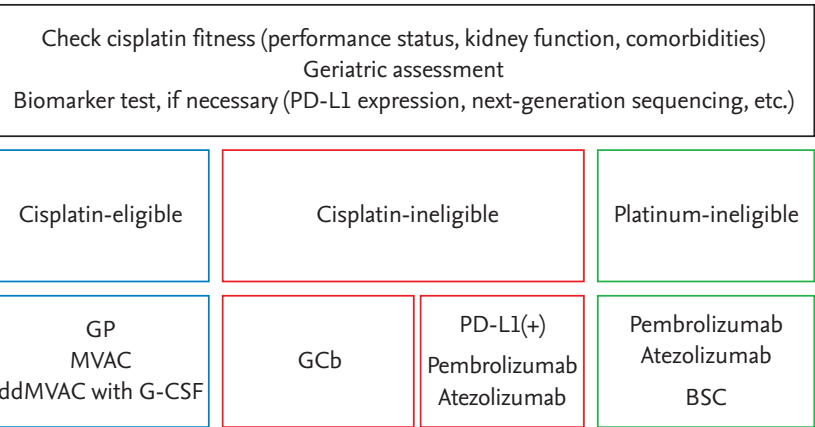

Figure 3. Suggestions for first-line treatment alternatives for patients with metastatic urothelial cancer. PD-L1, programmed death ligand 1; GP, gemcitabine plus cisplatin; MVAC, methotrexate, vinblastine, adriamycin, and cisplatin; ddMVAC, dose-dense MVAC; G-CSF, granulocyte colony-stimulating factor; $\mathrm{GCb}$, gemcitabine plus carboplatin; BSC, best supportive care.

The above models are all based on data from clinical trials of cytotoxic chemotherapy; therefore, whether they can be applied for ICI is not certain. There is no well-validated prognostic model specific to ICI.

\section{First-line treatment for patients with $\mathrm{mUC}$}

As of 2020, cisplatin-based combination chemotherapy remains the standard treatment for cisplatin-eligible patients (Fig. 3). Both GP and MVAC can be used, 


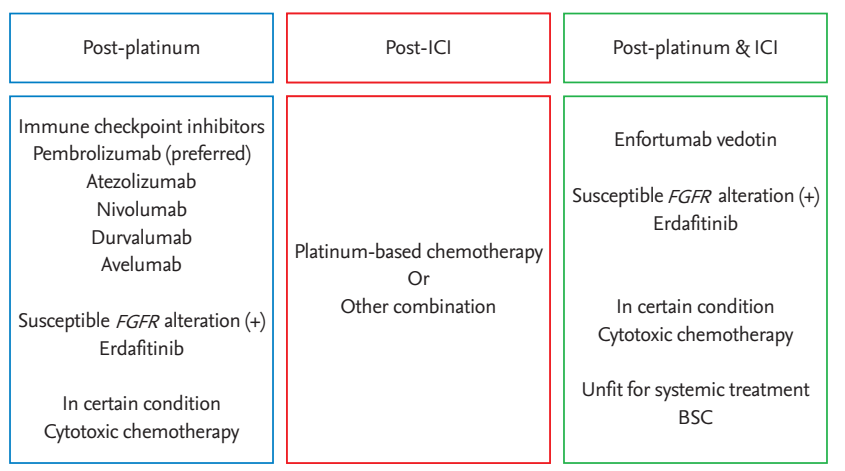

Figure 4. Suggestions for second- or later-line treatment alternatives for patients with metastatic urothelial cancer. ICI, immune checkpoint inhibitor; FGFR, fibroblast growth factor receptor; BSC, best supportive care.

and ddMVAC with prophylactic G-CSF can also be used $[4,5,7,8]$. All of these regimens are deemed to have similar efficacy, but their toxicity profiles differ from one another. For cisplatin-ineligible patients, there is no universally accepted standard treatment. $\mathrm{GCb}$ has been the most widely used regimen [32]. Considering longer survival in atezolizumab and pembrolizumab arms among cisplatin-ineligible patients compared to the historical control in IMvigor210 and KEYNOTE-052 trials [65,66], and numerically longer survival of atezolizumab monotherapy arm compared to the chemotherapy arm in interim results of the IMvigorizo trial [69], ICIs can also be used in only PD-Lı high patients [68].

When platinum cannot be used (platinum-ineligible), ICIs can be used regardless of PD-L1 expression, even though the criteria for "platinum-ineligibility" are not yet well defined [140]. If ICIs are not available or patients have conditions to avoid ICIs, single-agent gemcitabine can be considered, although there is no convincing evidence. If patients cannot tolerate any systemic treatment, offer BSC only.

\section{Second- or later-line treatment}

For patients who progressed after platinum-based chemotherapy, ICIs are the standard treatment option (Fig. 4) [50-54]. Among FDA-approved ICIs (atezolizumab, avelumab, durvalumab, nivolumab, and pembrolizum$\mathrm{ab}$ ), pembrolizumab is the preferred choice based on the KEYNOTE-045 trial [50]. For patients whose tumors have susceptible FGFR2 or 3 mutations, erdafitinib can be considered [8o].
When patients progress after first-line ICI, cytotoxic chemotherapy can be administered. There are no prospective trial data in this situation, but platinum-based chemotherapies would be preferred, if we take account of their efficacy in a first-line setting. Similar to firstline setting, the regimen can be chosen according to cisplatin-eligibility.

For patients who failed both platinum and ICI, enfortumab vedotin was proven effective [118]. Erdafitinib can also be used if there are susceptible FGFR alterations. Under certain conditions, when all available treatment fails or novel drugs are unavailable, classical cytotoxic chemotherapy (paclitaxel, docetaxel, pemetrexed, or vinflunine) might be considered for palliation.

At any point of time, if the general condition deteriorates or the patient seems not to tolerate systemic treatment, offer BSC only.

\section{CONCLUSIONS}

Systemic treatment of mUC has been stagnant for many decades, but revolutionary breakthroughs have now occurred. Guidelines have now included ICIs (atezolizumab, avelumab, durvalumab, nivolumab, and pembrolizumab), FGFR3 inhibitors (erdafitinib), and ADCs (enfortumab vedotin) in treatment protocols. Furthermore, multiple clinical trials, including but not limited to those mentioned above, are ongoing. We believe that the prospect of the therapeutic landscape is promising with this armamentarium. However, great effort is needed to fill the gap between treatment guidelines and real-world practice, as patients with mUC are more likely to be elderly and comorbid than those with other cancers, and there are growing concerns about the affordability and availability of novel treatments.

\section{Conflict of interest}

No potential conflict of interest relevant to this article was reported.

\section{REFERENCES}

1. Bray F, Ferlay J, Soerjomataram I, Siegel RL, Torre LA, Jemal A. Global cancer statistics 2018: GLOBOCAN esti- 
mates of incidence and mortality worldwide for 36 cancers in 185 countries. CA Cancer J Clin 2018;68:394-424.

2. Royce TJ, Lin CC, Gray PJ, Shipley WU, Jemal A, Efstathiou JA. Clinical characteristics and outcomes of nonurothelial cell carcinoma of the bladder: results from the National Cancer Data Base. Urol Oncol 2018;36:78.e178.e12.

3. Cumberbatch MGK, Jubber I, Black PC, et al. Epidemiology of bladder cancer: a systematic review and contemporary update of risk factors in 2018. Eur Urol 2018;74:784795 .

4. Sternberg CN, de Mulder P, Schornagel JH, et al. Seven year update of an EORTC phase III trial of high-dose intensity M-VAC chemotherapy and G-CSF versus classic M-VAC in advanced urothelial tract tumours. Eur J Cancer 2006;42:50-54.

5. von der Maase H, Sengelov L, Roberts JT, et al. Long-term survival results of a randomized trial comparing gemcitabine plus cisplatin, with methotrexate, vinblastine, doxorubicin, plus cisplatin in patients with bladder cancer. J Clin Oncol 2005;23:4602-4608.

6. Abdollah F, Gandaglia G, Thuret R, et al. Incidence, survival and mortality rates of stage-specific bladder cancer in United States: a trend analysis. Cancer Epidemiol 2013:37:219-225.

7. Sternberg CN, de Mulder PH, Schornagel JH, et al. Randomized phase III trial of high-dose-intensity methotrexate, vinblastine, doxorubicin, and cisplatin (MVAC) chemotherapy and recombinant human granulocyte colony-stimulating factor versus classic MVAC in advanced urothelial tract tumors: European Organization for Research and Treatment of Cancer Protocol no. 30924. J Clin Oncol 2001;19:2638-2646.

8. von der Maase H, Hansen SW, Roberts JT, et al. Gemcitabine and cisplatin versus methotrexate, vinblastine, doxorubicin, and cisplatin in advanced or metastatic bladder cancer: results of a large, randomized, multinational, multicenter, phase III study. J Clin Oncol 2000;18:3068-3077.

9. Oliver RT, Newlands ES, Wiltshaw E, Malpas JS. A phase 2 study of Cis-platinum in patients with recurrent bladder carcinoma. The London and Oxford Co-operative Urological Cancer Group. Br J Urol 1981;53:444-447.

10. Rossof AH, Talley RW, Stephens R, et al. Phase II evaluation of cis-dichlorodiammineplatinum(II) in advanced malignancies of the genitourinary and gynecologic or- gans: a Southwest Oncology Group Study. Cancer Treat Rep 1979;63:1557-1564.

11. Sternberg CN, Yagoda A, Scher HI, et al. Methotrexate, vinblastine, doxorubicin, and cisplatin for advanced transitional cell carcinoma of the urothelium. Efficacy and patterns of response and relapse. Cancer 1989;64:24482458.

12. Loehrer PJ Sr, Einhorn LH, Elson PJ, et al. A randomized comparison of cisplatin alone or in combination with methotrexate, vinblastine, and doxorubicin in patients with metastatic urothelial carcinoma: a cooperative group study. J Clin Oncol 1992;10:1066-1073.

13. Logothetis CJ, Dexeus FH, Finn L, et al. A prospective randomized trial comparing MVAC and CISCA chemotherapy for patients with metastatic urothelial tumors. J Clin Oncol 1990;8:1050-1055.

14. Stadler WM, Kuzel T, Roth B, Raghavan D, Dorr FA. Phase II study of single-agent gemcitabine in previously untreated patients with metastatic urothelial cancer. J Clin Oncol 1997;15:3394-3398.

15. Moore MJ, Winquist EW, Murray N, et al. Gemcitabine plus cisplatin, an active regimen in advanced urothelial cancer: a phase II trial of the National Cancer Institute of Canada Clinical Trials Group. J Clin Oncol 1999;17:28762881.

16. Soto Parra H, Cavina R, Latteri F, et al. Three-week versus four-week schedule of cisplatin and gemcitabine: results of a randomized phase II study. Ann Oncol 2002;13:10801086.

17. Als AB, Sengelov L, Von Der Maase H. Gemcitabine and cisplatin in locally advanced and metastatic bladder cancer; 3- or 4-week schedule? Acta Oncol 2008;47:110-119.

18. Roth BJ, Dreicer R, Einhorn LH, et al. Significant activity of paclitaxel in advanced transitional-cell carcinoma of the urothelium: a phase II trial of the Eastern Cooperative Oncology Group. J Clin Oncol 1994;12:2264-2270.

19. de Wit R, Kruit WH, Stoter G, de Boer M, Kerger J, Verweij J. Docetaxel (Taxotere): an active agent in metastatic urothelial cancer; results of a phase II study in non-chemotherapy-pretreated patients. Br J Cancer 1998;78:13421345 .

20. Bamias A, Aravantinos G, Deliveliotis C, et al. Docetaxel and cisplatin with granulocyte colony-stimulating factor (G-CSF) versus MVAC with G-CSF in advanced urothelial carcinoma: a multicenter, randomized, phase III study from the Hellenic Cooperative Oncology Group. J Clin 
Oncol 2004;22:220-228.

21. Sternberg CN, Skoneczna IA, Castellano D, et al. Larotaxel with cisplatin in the first-line treatment of locally advanced/metastatic urothelial tract or bladder cancer: a randomized, active-controlled, phase III trial (CILAB). Oncology 2013;85:208-215.

22. Bellmunt J, von der Maase H, Mead GM, et al. Randomized phase III study comparing paclitaxel/cisplatin/ gemcitabine and gemcitabine/cisplatin in patients with locally advanced or metastatic urothelial cancer without prior systemic therapy: EORTC Intergroup Study 30987. J Clin Oncol 2012;30:1107-1113.

23. Bellmunt J, Orsola A, Leow JJ, et al. Bladder cancer: ESMO Practice Guidelines for diagnosis, treatment and follow-up. Ann Oncol 2014;25 Suppl 3:iii4o-iii48.

24. National Comprehensive Cancer Network. Bladder cancer (version 3.2020) [Internet]. Plymouth Meeting (PA): NCCN, 2020 [cited 2020 Jun 26]. Available from: https:// www.nccn.org/professionals/physician_gls/pdf/bladder. pdf.

25. Witjes JA, Bruins HM, Cathomas R, et al. Guidelines on muscle-invasive and metastatic bladder cancer [Internet]. Arnhem (NL): EAU Guidelines Office, c2020 [cited 2020 Jun 26]. Available from: https://uroweb.org/guideline/ bladder-cancer-muscle-invasive-and-metastatic/.

26. Muggia FM, Bonetti A, Hoeschele JD, Rozencweig M, Howell SB. Platinum antitumor complexes: 50 years since Barnett Rosenberg's discovery. J Clin Oncol 2015;33:42194226.

27. Petrioli R, Frediani B, Manganelli A, et al. Comparison between a cisplatin-containing regimen and a carboplatin-containing regimen for recurrent or metastatic bladder cancer patients. A randomized phase II study. Cancer 1996;77:344-351.

28. Dogliotti L, Carteni G, Siena S, et al. Gemcitabine plus cisplatin versus gemcitabine plus carboplatin as first-line chemotherapy in advanced transitional cell carcinoma of the urothelium: results of a randomized phase 2 trial. Eur Urol 2007;52:134-141.

29. Galsky MD, Chen GJ, Oh WK, et al. Comparative effectiveness of cisplatin-based and carboplatin-based chemotherapy for treatment of advanced urothelial carcinoma. Ann Oncol 2012;23:406-410.

30. Galsky MD, Hahn NM, Rosenberg J, et al. A consensus definition of patients with metastatic urothelial carcinoma who are unfit for cisplatin-based chemotherapy. Lan- cet Oncol 2011;12:211-214.

31. Galsky MD, Hahn NM, Rosenberg J, et al. Treatment of patients with metastatic urothelial cancer "unfit" for cisplatin-based chemotherapy. J Clin Oncol 2011;29:24322438.

32. De Santis M, Bellmunt J, Mead G, et al. Randomized phase II/III trial assessing gemcitabine/carboplatin and methotrexate/carboplatin/vinblastine in patients with advanced urothelial cancer who are unfit for cisplatin-based chemotherapy: EORTC study 30986. J Clin Oncol 2012;30:191-199.

33. Holmsten K, Jensen NV, Mouritsen LS, et al. Vinflunine/ gemcitabine versus carboplatin/gemcitabine as first-line treatment in cisplatin-ineligible patients with advanced urothelial carcinoma: a randomised phase II trial (VINGEM). Eur J Cancer 2020;127:173-182.

34. Park I, Kim BS, Lim HY, et al. Gemcitabine plus carboplatin versus gemcitabine plus oxaliplatin in cisplatin-unfit patients with advanced urothelial carcinoma: a randomised phase II study (COACH, KCSG GU10-16). Eur J Cancer 2020;127:183-190.

35. De Santis M, Wiechno PJ, Bellmunt J, et al. Vinflunine-gemcitabine versus vinflunine-carboplatin as first-line chemotherapy in cisplatin-unfit patients with advanced urothelial carcinoma: results of an international randomized phase II trial (JASINT1). Ann Oncol 2016;27:449-454.

36. Bellmunt J, Kerst JM, Vazquez F, et al. A randomized phase II/III study of cabazitaxel versus vinflunine in metastatic or locally advanced transitional cell carcinoma of the urothelium (SECAVIN). Ann Oncol 2017;28:1517-1522.

37. Papamichael D, Gallagher CJ, Oliver RT, Johnson PW, Waxman J. Phase II study of paclitaxel in pretreated patients with locally advanced/metastatic cancer of the bladder and ureter. Br J Cancer 1997;75:606-607.

38. Joly F, Houede N, Noal S, et al. Do patients with advanced urothelial carcinoma benefit from weekly paclitaxel chemotherapy? A GETUG phase II study. Clin Genitourin Cancer 2009;7:E28-E33.

39. Beer TM, Goldman B, Nichols CR, et al. Southwest Oncology Group phase II study of irinotecan in patients with advanced transitional cell carcinoma of the urothelium that progressed after platinum-based chemotherapy. Clin Genitourin Cancer 2008;6:36-39.

40. Lee JL, Ahn JH, Park SH, et al. Phase II study of a cremophor-free, polymeric micelle formulation of paclitaxel for 
patients with advanced urothelial cancer previously treated with gemcitabine and platinum. Invest New Drugs 2012;30:1984-1990.

41. Galsky MD, Mironov S, Iasonos A, Scattergood J, Boyle MG, Bajorin DF. Phase II trial of pemetrexed as second-line therapy in patients with metastatic urothelial carcinoma. Invest New Drugs 2007;25:265-270.

42. Witte RS, Manola J, Burch PA, Kuzel T, Weinshel EL, Loehrer PJ Sr. Topotecan in previously treated advanced urothelial carcinoma: an ECOG phase II trial. Invest New Drugs 1998;16:191-195.

43. Witte RS, Elson P, Bono B, et al. Eastern Cooperative Oncology Group phase II trial of ifosfamide in the treatment of previously treated advanced urothelial carcinoma. J Clin Oncol 1997;15:589-593.

44. Sweeney CJ, Roth BJ, Kabbinavar FF, et al. Phase II study of pemetrexed for second-line treatment of transitional cell cancer of the urothelium. J Clin Oncol 2006;24:34513457.

45. Vaughn DJ, Broome CM, Hussain M, Gutheil JC, Markowitz AB. Phase II trial of weekly paclitaxel in patients with previously treated advanced urothelial cancer. J Clin Oncol 2002;20:937-940.

46. Ko YJ, Canil CM, Mukherjee SD, et al. Nanoparticle albumin-bound paclitaxel for second-line treatment of metastatic urothelial carcinoma: a single group, multicentre, phase 2 study. Lancet Oncol 2013;14:769-776.

47. Dreicer R, Li S, Manola J, et al. Phase 2 trial of epothilone $\mathrm{B}$ analog BMS-247550 (ixabepilone) in advanced carcinoma of the urothelium (E380o): a trial of the Eastern Cooperative Oncology Group. Cancer 2007;110:759-763.

48. Bellmunt J, Fougeray R, Rosenberg JE, et al. Long-term survival results of a randomized phase III trial of vinflunine plus best supportive care versus best supportive care alone in advanced urothelial carcinoma patients after failure of platinum-based chemotherapy. Ann Oncol 2013;24:1466-1472.

49. Bellmunt J, Theodore C, Demkov T, et al. Phase III trial of vinflunine plus best supportive care compared with best supportive care alone after a platinum-containing regimen in patients with advanced transitional cell carcinoma of the urothelial tract. J Clin Oncol 2009;27:4454-4461.

50. Bellmunt J, de Wit R, Vaughn DJ, et al. Pembrolizumab as second-line therapy for advanced urothelial carcinoma. N Engl J Med 2017;376:1015-1026.

51. Powles T, Duran I, van der Heijden MS, et al. Atezolizum- ab versus chemotherapy in patients with platinum-treated locally advanced or metastatic urothelial carcinoma (IMvigor211): a multicentre, open-label, phase 3 randomised controlled trial. Lancet 2018;391:748-757.

52. Sharma P, Retz M, Siefker-Radtke A, et al. Nivolumab in metastatic urothelial carcinoma after platinum therapy (CheckMate 275): a multicentre, single-arm, phase 2 trial. Lancet Oncol 2017;18:312-322.

53. Powles T, O’Donnell PH, Massard C, et al. Efficacy and safety of durvalumab in locally advanced or metastatic urothelial carcinoma: updated results from a phase $1 / 2$ open-label study. JAMA Oncol 2017;3:e172411.

54. Patel MR, Ellerton J, Infante JR, et al. Avelumab in metastatic urothelial carcinoma after platinum failure (JAVELIN Solid Tumor): pooled results from two expansion cohorts of an open-label, phase 1 trial. Lancet Oncol 2018;19:51-64.

55. Fradet Y, Bellmunt J, Vaughn DJ, et al. Randomized phase III KEYNOTE-045 trial of pembrolizumab versus paclitaxel, docetaxel, or vinflunine in recurrent advanced urothelial cancer: results of $>2$ years of follow-up. Ann Oncol 2019;30:970-976.

56. Van der Heijden MS, Loriot Y, Duran I, et al. 918P: Atezolizumab (atezo) vs chemotherapy (chemo) in patients (pts) with platinum-treated locally advanced or metastatic urothelial carcinoma (mUC): a long-term overall survival (OS) and safety update from the phase III IMvigor211 study. Ann Oncol 2019;30(Suppl 5):v365-v366.

57. Hur JY, Kim Y, Kwon GY, et al. Atezolizumab in patients with pretreated urothelial cancer: a Korean single-center, retrospective study. Cancer Res Treat 2019;51:1269-1274.

58. Sternberg CN, Loriot Y, James N, et al. Primary results from SAUL, a multinational single-arm safety study of atezolizumab therapy for locally advanced or metastatic urothelial or nonurothelial carcinoma of the urinary tract. Eur Urol 2019;76:73-81.

59. Brahmer JR, Lacchetti C, Schneider BJ, et al. Management of immune-related adverse events in patients treated with immune checkpoint inhibitor therapy: American Society of Clinical Oncology Clinical Practice Guideline. J Clin Oncol 2018;36:1714-1768.

6o. Martins F, Sofiya L, Sykiotis GP, et al. Adverse effects of immune-checkpoint inhibitors: epidemiology, management and surveillance. Nat Rev Clin Oncol 2019;16:563580.

61. Borcoman E, Kanjanapan Y, Champiat S, et al. Novel 
patterns of response under immunotherapy. Ann Oncol 2019;30:385-396.

62. Hwang I, Park I, Yoon SK, Lee JL. Hyperprogressive disease in patients with urothelial carcinoma or renal cell carcinoma treated with PD-1/PD-L1 inhibitors. Clin Genitourin Cancer 2020;18:e122-e133.

63. Soria F, Beleni AI, D'Andrea D, et al. Pseudoprogression and hyperprogression during immune checkpoint inhibitor therapy for urothelial and kidney cancer. World J Urol 2018;36:1703-1709.

64. Powles T, Park SH, Voog E, et al. Maintenance avelum$\mathrm{ab}+$ best supportive care (BSC) versus BSC alone after platinum-based first-line (1L) chemotherapy in advanced urothelial carcinoma (UC): JAVELIN Bladder 100 phase III interim analysis. J Clin Oncol 2020;38(18 Suppl):LBA1.

65. Balar AV, Galsky MD, Rosenberg JE, et al. Atezolizumab as first-line treatment in cisplatin-ineligible patients with locally advanced and metastatic urothelial carcinoma: a single-arm, multicentre, phase 2 trial. Lancet 2017;389:6776.

66. Balar AV, Castellano D, O'Donnell PH, et al. First-line pembrolizumab in cisplatin-ineligible patients with locally advanced and unresectable or metastatic urothelial cancer (KEYNOTE-052): a multicentre, single-arm, phase 2 study. Lancet Oncol 2017;18:1483-1492.

67. O'Donnell PH, Balar AV, Vuky J, et al. KEYNOTE-052: Phase 2 study evaluating first-line pembrolizumab (pembro) in cisplatin-ineligible advanced urothelial cancer (UC): updated response and survival results. J Clin Oncol 2019;37(15 Suppl):4546.

68. Suzman DL, Agrawal S, Ning YM, et al. FDA approval summary: atezolizumab or pembrolizumab for the treatment of patients with advanced urothelial carcinoma ineligible for cisplatin-containing chemotherapy. Oncologist 2019;24:563-569.

69. Galsky MD, Arija JAA, Bamias A, et al. Atezolizumab with or without chemotherapy in metastatic urothelial cancer (IMvigor130): a multicentre, randomised, placebo-controlled phase 3 trial. Lancet 2020;395:1547-1557.

70. AstraZeneca. Update on phase III DANUBE trial for imfinzi and tremelimumab in unresectable, stage IV bladder cancer [Internet]. Cambridge (UK): AstraZeneca, 2020 [cited 2020 Jun 26]. Available from: https://www.astrazeneca.com/media-centre/press-releases/2020/update-onphase-iii-danube-trial-for-imfinzi-and-tremelimumabin-unresectable-stage-iv-bladder-cancer-o6032020.html.
71. Iyer G, Al-Ahmadie H, Schultz N, et al. Prevalence and co-occurrence of actionable genomic alterations in highgrade bladder cancer. J Clin Oncol 2013;31:3133-3140.

72. Cancer Genome Atlas Research Network. Comprehensive molecular characterization of urothelial bladder carcinoma. Nature 2014;507:315-322.

73. Ross JS, Wang K, Al-Rohil RN, et al. Advanced urothelial carcinoma: next-generation sequencing reveals diverse genomic alterations and targets of therapy. Mod Pathol 2014;27:271-280.

74. Robertson AG, Kim J, Al-Ahmadie H, et al. Comprehensive molecular characterization of muscle-invasive bladder cancer. Cell 2017;171:540-556.

75. Sethakorn N, O’Donnell PH. Spectrum of genomic alterations in FGFR3: current appraisal of the potential role of $\mathrm{FGFR}_{3}$ in advanced urothelial carcinoma. BJU Int 2016;118:681-691.

76. Hurst CD, Knowles MA. Mutational landscape of non-muscle-invasive bladder cancer. Urol Oncol $2018 \mathrm{Nov}$ 13 [Epub]. https://doi.org/10.1016/j.urolonc.2018.10.015.

77. Guancial EA, Werner L, Bellmunt J, et al. FGFR3 expression in primary and metastatic urothelial carcinoma of the bladder. Cancer Med 2014;3:835-844.

78. Dip N, Reis ST, Viana NI, et al. MiRNA in bladder carcinogenesis: a review. World J Clin Urol 2014;3:238-248.

79. Milowsky MI, Dittrich C, Duran I, et al. Phase 2 trial of dovitinib in patients with progressive FGFR3-mutated or $\mathrm{FGFR}_{3}$ wild-type advanced urothelial carcinoma. Eur J Cancer 2014;50:3145-3152.

8o. Loriot Y, Necchi A, Park SH, et al. Erdafitinib in locally advanced or metastatic urothelial carcinoma. N Engl J Med 2019;381:338-348.

81. Schuler M, Cho BC, Sayehli CM, et al. Rogaratinib in patients with advanced cancers selected by FGFR mRNA expression: a phase 1 dose-escalation and dose-expansion study. Lancet Oncol 2019;20:1454-1466.

82. Quinn DI, Petrylak DP, Bellmunt J, et al. FORT-1: Phase II/III study of rogaratinib versus chemotherapy (CT) in patients (pts) with locally advanced or metastatic urothelial carcinoma (UC) selected based on FGFR1/3 mRNA expression. J Clin Oncol 2020;38(6 Suppl):489.

83. Pal SK, Rosenberg JE, Hoffman-Censits JH, et al. Efficacy of BGJ398, a fibroblast growth factor receptor 1-3 inhibitor, in patients with previously treated advanced urothelial carcinoma with FGFR 3 alterations. Cancer Discov 2018;8:812-821. 
84. Necchi A, Pouessel D, Leibowitz-Amit R, et al. 90oP: Interim results of fight-201, a phase II, open-label, multicenter study of INCBo54828 in patients (pts) with metastatic or surgically unresectable urothelial carcinoma (UC) harboring fibroblast growth factor (FGF)/FGF receptor (FGFR) genetic alterations (GA). Ann Oncol 2018;29(Suppl 8):viiiz19-viiiz2o.

85. Mellado B, Castellano DE, Pang $\mathrm{S}$, et al. Interim analysis of the fierce-21 phase 2 ( $\left.\mathrm{P}_{2}\right)$ study of vofatamab (B-701), a selective inhibitor of $\mathrm{FGFR}_{3}$, as salvage therapy in metastatic urothelial carcinoma (mUC). J Clin Oncol 2019;37(15 Suppl):4547.

86. Sweis RF, Spranger S, Bao R, et al. Molecular drivers of the non-t-cell-inflamed tumor microenvironment in urothelial bladder cancer. Cancer Immunol Res 2016;4:563-568.

87. Palakurthi S, Kuraguchi M, Zacharek SJ, et al. The combined effect of FGFR inhibition and PD-1 blockade promotes tumor-intrinsic induction of antitumor immunity. Cancer Immunol Res 2019;7:1457-1471.

88. Siefker-Radtke AO, Currie G, Abella E, et al. FIERCE-22: Clinical activity of vofatamab (V) a FGFR3 selective inhibitor in combination with pembrolizumab (P) in WT metastatic urothelial carcinoma, preliminary analysis. J Clin Oncol 2019;37(15 Suppl):4511.

89. Powles T, Balar A, Gravis G, et al. 902O: An adaptive, biomarker directed platform study in metastatic urothelial cancer (BISCAY) with durvalumab in combination with targeted therapies. Ann Oncol 2019;30(Suppl 5):v356-v357.

90. Grivas PD, Day M, Hussain M. Urothelial carcinomas: a focus on human epidermal receptors signaling. Am J Transl Res 2011;3:362-373.

91. Petrylak DP, Tangen CM, Van Veldhuizen PJ Jr, et al. Results of the Southwest Oncology Group phase II evaluation (study So031) of ZD1839 for advanced transitional cell carcinoma of the urothelium. BJU Int 2010;105:317-321.

92. Philips GK, Halabi S, Sanford BL, Bajorin D, Small EJ; Cancer and Leukemia Group B. A phase II trial of cisplatin $(\mathrm{C})$, gemcitabine $(\mathrm{G})$ and gefitinib for advanced urothelial tract carcinoma: results of Cancer and Leukemia Group B (CALGB) 90102. Ann Oncol 2009;20:10741079.

93. Wong YN, Litwin S, Vaughn D, et al. Phase II trial of cetuximab with or without paclitaxel in patients with advanced urothelial tract carcinoma. J Clin Oncol 2012;30:3545-3551.

94. Hussain M, Daignault S, Agarwal N, et al. A randomized phase 2 trial of gemcitabine/cisplatin with or without cetuximab in patients with advanced urothelial carcinoma. Cancer 2014;120:2684-2693.

95. Wülfing C, Machiels JP, Richel DJ, et al. A single-arm, multicenter, open-label phase 2 study of lapatinib as the second-line treatment of patients with locally advanced or metastatic transitional cell carcinoma. Cancer 2009;115:2881-2890.

96. Tang S, Dorff TB, Tsao-Wei DD, et al. Single arm phase II study of docetaxel and lapatinib in metastatic urothelial cancer: USC trial 4B-10-4. J Clin Oncol 2016;34(2 Suppl):424.

97. Cerbone L, Sternberg CN, Sengelov L, et al. Results from a phase I study of lapatinib with gemcitabine and cisplatin in advanced or metastatic bladder cancer: EORTC trial 30061. Oncology 2016;90:21-28.

98. Powles T, Huddart RA, Elliott T, et al. Phase III, double-blind, randomized trial that compared maintenance lapatinib versus placebo after first-line chemotherapy in patients with human epidermal growth factor receptor 1/2-positive metastatic bladder cancer. J Clin Oncol 2017;35:48-55.

99. Choudhury NJ, Campanile A, Antic T, et al. Afatinib activity in platinum-refractory metastatic urothelial carcinoma in patients with ERBB alterations. J Clin Oncol 2016;34:2165-2171.

100. Hussain MH, MacVicar GR, Petrylak DP, et al. Trastuzumab, paclitaxel, carboplatin, and gemcitabine in advanced human epidermal growth factor receptor-2/neu-positive urothelial carcinoma: results of a multicenter phase II National Cancer Institute trial. J Clin Oncol 2007;25:22182224.

101. Oudard S, Culine S, Vano Y, et al. Multicentre randomised phase II trial of gemcitabine+platinum, with or without trastuzumab, in advanced or metastatic urothelial carcinoma overexpressing Her2. Eur J Cancer 2015;51:45-54.

102. Bryce AH, Kurzrock R, Meric-Bernstam F, et al. Pertuzumab plus trastuzumab for HER2-positive metastatic urothelial cancer (mUC): preliminary data from MyPathway. J Clin Oncol 2017;35(6 Suppl):348.

103. Fus LP, Gornicka B. Role of angiogenesis in urothelial bladder carcinoma. Cent European J Urol 2016;69:258263.

104. Dreicer R, Li H, Stein M, et al. Phase 2 trial of sorafenib in patients with advanced urothelial cancer: a trial of the Eastern Cooperative Oncology Group. Cancer 2009;115:4090-4095. 
105. Bellmunt J, Gonzalez-Larriba JL, Prior C, et al. Phase II study of sunitinib as first-line treatment of urothelial cancer patients ineligible to receive cisplatin-based chemotherapy: baseline interleukin-8 and tumor contrast enhancement as potential predictive factors of activity. Ann Oncol 2011;22:2646-2653.

106. Necchi A, Mariani L, Zaffaroni N, et al. Pazopanib in advanced and platinum-resistant urothelial cancer: an open-label, single group, phase 2 trial. Lancet Oncol 2012;13:810-816.

107. Jones RJ, Hussain SA, Protheroe AS, et al. Randomized phase II study investigating pazopanib versus weekly paclitaxel in relapsed or progressive urothelial cancer. J Clin Oncol 2017;35:1770-1777.

108. Apolo AB, Parnes HL, Francis DC, et al. A phase II study of cabozantinib in patients (pts) with relapsed or refractory metastatic urothelial carcinoma (mUC). J Clin Oncol 2016;34(15 Suppl):4534.

109. Naik G, Vaishampayan UN, Nabell L, et al. A phase II trial of regorafenib for advanced urothelial cancer (aUC) following prior chemotherapy. J Clin Oncol 2020;38(6 Supp1):498.

110. Galsky MD, Hahn NM, Powles T, et al. Gemcitabine, cisplatin, and sunitinib for metastatic urothelial carcinoma and as preoperative therapy for muscle-invasive bladder cancer. Clin Genitourin Cancer 2013;11:175-181.

111. Choueiri TK, Ross RW, Jacobus S, et al. Double-blind, randomized trial of docetaxel plus vandetanib versus docetaxel plus placebo in platinum-pretreated metastatic urothelial cancer. J Clin Oncol 2012;30:507-512.

112. Narayanan S, Lam A, Vaishampayan U, et al. Phase II study of pazopanib and paclitaxel in patients with refractory urothelial cancer. Clin Genitourin Cancer 2016;14:432-437.

113. Petrylak DP, de Wit R, Chi KN, et al. Ramucirumab plus docetaxel versus placebo plus docetaxel in patients with locally advanced or metastatic urothelial carcinoma after platinum-based therapy (RANGE): a randomised, double-blind, phase 3 trial. Lancet 2017;390:2266-2277.

114. Petrylak DP, de Wit R, Chi KN, et al. Ramucirumab plus docetaxel versus placebo plus docetaxel in patients with locally advanced or metastatic urothelial carcinoma after platinum-based therapy (RANGE): overall survival and updated results of a randomised, double-blind, phase 3 trial. Lancet Oncol 2020;21:105-120.

115. Rosenberg JE, Ballman KV, Halabi S, et al. CALGB 90601 (Alliance): randomized, double-blind, placebo-controlled phase III trial comparing gemcitabine and cisplatin with bevacizumab or placebo in patients with metastatic urothelial carcinoma. J Clin Oncol 2019;37(15 Suppl):4503.

116. Zhu N, Weng S, Wang J, et al. Preclinical rationale and clinical efficacy of antiangiogenic therapy and immune checkpoint blockade combination therapy in urogenital tumors. J Cancer Res Clin Oncol 2019;145:3021-3036.

117. Birrer MJ, Moore KN, Betella I, Bates RC. Antibody-drug conjugate-based therapeutics: state of the science. J Natl Cancer Inst 2019;111:538-549.

118. Rosenberg JE, O’Donnell PH, Balar AV, et al. Pivotal trial of enfortumab vedotin in urothelial carcinoma after platinum and anti-programmed death 1/programmed death ligand 1 therapy. J Clin Oncol 2019;37:2592-2600.

119. Mandai K, Rikitake Y, Mori M, Takai Y. Nectins and nectin-like molecules in development and disease. Curr Top Dev Biol 2015;112:197-231.

120. Challita-Eid PM, Satpayev D, Yang P, et al. Enfortumab vedotin antibody-drug conjugate targeting nectin- 4 is a highly potent therapeutic agent in multiple preclinical cancer models. Cancer Res 2016;76:3003-3013.

121. Rosenberg JE, Flaig TW, Friedlander TW, et al. Study EV103: preliminary durability results of enfortumab vedotin plus pembrolizumab for locally advanced or metastatic urothelial carcinoma. J Clin Oncol 2020;38(6 Suppl):441.

122. Trerotola M, Cantanelli P, Guerra E, et al. Upregulation of Trop-2 quantitatively stimulates human cancer growth. Oncogene 2013;32:222-233.

123. Avellini C, Licini C, Lazzarini R, et al. The trophoblast cell surface antigen 2 and miR-125b axis in urothelial bladder cancer. Oncotarget 2017;8:58642-58653.

124. Tagawa ST, Faltas BM, Lam ET, et al. Sacituzumab govitecan (IMMU-132) in patients with previously treated metastatic urothelial cancer (mUC): results from a phase I/II study. J Clin Oncol 2019;37(7 Suppl):354.

125. Tagawa ST, Balar A, Petrylak DP, et al. LBA55: Initial results from TROPHY-U-o1: A phase II open-label study of sacituzumab govitecan in patients (Pts) with metastatic urothelial cancer (mUC) after failure of platinum-based regimens (PLT) or immunotherapy. Ann Oncol 2019;30(Suppl 5):v89o-v891.

126. Yao X, Jiang J, Wang X, et al. A novel humanized anti-HER2 antibody conjugated with MMAE exerts potent anti-tumor activity. Breast Cancer Res Treat 2015;153:123133.

127. Sheng X, Zhou AP, Yao X, et al. A phase II study of RC48- 
ADC in HER2-positive patients with locally advanced or metastatic urothelial carcinoma. J Clin Oncol 2019;37(15 Suppl):4509.

128. ClinicalTrials.gov. Trastuzumab deruxtecan with nivolum$\mathrm{ab}$ in advanced breast and urothelial cancer [Internet]. Bethesda (MD): ClinicalTrials.gov, 2020 [cited 2020 Jun 26]. Available from: https://ClinicalTrials.gov/show/ NCT03523572.

129. Al-Husseini MJ, Kunbaz A, Saad AM, et al. Trends in the incidence and mortality of transitional cell carcinoma of the bladder for the last four decades in the USA: a SEERbased analysis. BMC Cancer 2019;19:46.

130. Repetto L. Greater risks of chemotherapy toxicity in elderly patients with cancer. J Support Oncol 2003;1(4 Suppl 2):18-24.

131. Galsky MD, Pal SK, Lin SW, et al. Real-world effectiveness of chemotherapy in elderly patients with metastatic bladder cancer in the United States. Bladder Cancer 2018;4:227-238.

132. Mohile SG, Stadler WM. Do elderly patients have a worse outcome in response to platinum-based therapy for metastatic urothelial cancer? Nat Clin Pract Oncol 2005;2:392393 .

133. Hurria A, Togawa K, Mohile SG, et al. Predicting chemotherapy toxicity in older adults with cancer: a prospective multicenter study. J Clin Oncol 2011;29:3457-3465.
134. Garg T, Young AJ, Kost KA, et al. Burden of multiple chronic conditions among patients with urological cancer. J Urol 2018;199:543-550.

135. Lee L, Cheung WY, Atkinson E, Krzyzanowska MK. Impact of comorbidity on chemotherapy use and outcomes in solid tumors: a systematic review. J Clin Oncol 2011;29:106-117.

136. Klapheke A, Yap SA, Pan K, Cress RD. Sociodemographic disparities in chemotherapy treatment and impact on survival among patients with metastatic bladder cancer. Urol Oncol 2018;36:308.e19-308.

137. Johnson DB, Sullivan RJ, Menzies AM. Immune checkpoint inhibitors in challenging populations. Cancer 2017;123:1904-1911.

138. Bajorin DF, Dodd PM, Mazumdar M, et al. Long-term survival in metastatic transitional-cell carcinoma and prognostic factors predicting outcome of therapy. J Clin Oncol 1999;17:3173-3181.

139. Bellmunt J, Choueiri TK, Fougeray R, et al. Prognostic factors in patients with advanced transitional cell carcinoma of the urothelial tract experiencing treatment failure with platinum-containing regimens. J Clin Oncol 2010;28:1850-1855.

140. Gupta S, Sonpavde G, Grivas P, et al. Defining "platinum-ineligible" patients with metastatic urothelial cancer (mUC). J Clin Oncol 2019;37(7 Suppl):451. 